\title{
Experiencing Electric Vehicles: The Car as a Digital Platform
}

\author{
Thomas Lindgren \\ School of Information Technology, \\ Halmstad University, Sweden \\ thomas.lindgren@hh.se
}

\begin{abstract}
The transition towards electric vehicles $(E V) s$ is rapidly increasing, and the automotive industry has been busy exchanging to electric engines. However, electrification of cars is not only about hardware, but it also implies a digitalisation of cars. Charging and maintenance of EVS is enabled through digital platforms. Little is known of how this digitalisation of cars affect people's everyday lives. What happens when the car moves from being simply a machine that provides means for transport, but also becomes a power source and a sensor platform with computing capacity powered with AI driven technologies that is controlled via digital platforms? In this article, we present findings on how people experience and learn to live with EVs in their everyday life. We argue that ethnographic understandings of EVs as evolving digiphysical phenomena provide opportunities to foresight how to implement EVs from a people-centric perspective.
\end{abstract}

\section{Introduction}

After entering commercial markets in the first half of the decade, electric car sales have soared. According to the 'Global EV Outlook Report 2020' report, only about 17000 electric cars were on the world's roads in 2010. By 2019, that number had swelled to 7.2 million [1]. Ambitious policy announcements have set clear, long-term signals to the auto industry and consumers that support a continuing electric vehicle (EV) rollout in major vehicle markets in the coming years. However, little is yet known how this transition affects people's mobility patterns and use of the car, now that 'today's consumer profile in the electric car market is evolving from early adopters and technophile purchasers to mass adoption' [1]. This article will address this question, by example of our ethnographic investigations into how people adapt their everyday life to their EV and how this implies integrating a wide range of digital platforms into everyday logistics.

Research initiatives to understand people's attitudes to EVs can be found within the user experience (UX) research, where it focuses on driving related challenges, such as measuring rational behaviours of "range anxiety", lack of driving feedback or acceptance factors [2]. However, these initiatives tend to lean on traditions of how people rate fossil fuel cars, not taking into account how the drive and care of EVs are related to new emerging digital platforms for charging, energy management, payment, surveillance, or as mobile computing power units [3]. Thus, we argue for understanding the electrification of cars as enabling digi-physical experiences. The traditional automotive industry has also been mainly product focused in exchanging the combustion engine and fossil fuel for an electric engine and a battery [4]. For example, requirement specifications for EVs are based on the traditional fossil cars usage models and attribute parameters to meet the same range and driving performance. One such factor is batteries, and battery requirements are being articulated in terms of cost, energy density, power capability, life span, performance in different environments to optimize the driving range since it is said that this is one of the major customer concerns [5]. Critical voices have questioned that people's lack of interest, or resistance, in adopting EV's can solely be reduced by improving technical aspects of batteries in relation to driving range and subsequently mitigate psychological states of 'range anxiety' [6]. Thus, there is a need to understand EVs as part of a wider digital innovation agenda and to investigate what it means to live with an electrical car in a social and digitalised world.

In order to identify new challenges and opportunities EV's and related digital platforms could bring from an end user perspective, this paper proposes a design ethnographic approach to study people's anticipatory experiences and expectations of EV's. The approach in this paper is demonstrated through a study in how EVs integrate into people's 
everyday digital and physical lives. Hence, the RQ follows:

How can the impact digital platforms have on everyday life be understood through insights into people's everyday experiences and anticipations of electric vehicles? And what implications does these insights contribute to understandings of the digital transformation of car industries?

In this article we want to elaborate on how to approach the question of how digital platforms affect everyday life in relation to adoption of EVs, both how it become integrated with family logistics as well as how people anticipate future use when appropriating what is afforded through the digital platforms that our coupled with the use, maintenance and upgrade of the EV. We argue that ethnographic understandings of people's anticipatory experiences provide opportunities to foresight new challenges and opportunities that EVs and related digital platforms provide to people. These ethnographic foresights enable industry to better understand the transformative aspects of related digital platforms from an end user perspective in order to develop innovative and sustainable future mobility solutions.

\section{EV digital platform research}

Failing in understanding people's expectations and anticipations and controversies with technological innovations that straddle the physical and digital world through digital platforms may cause irreversible damage to the implementation of these technologies. One example is found in Google's investment in Google Glass as an extension of Google's digital platform into a wearable device [7]. Little was known about the serious immediate privacy concerns, the camera in the device made exposed to the people around a Google glass user. Some people thought the camera was always recording and quickly after the launch many users' experienced situations where it was seen inappropriate to wear them and they were called "Glassholes" (https://www.urbandictionary.com/define.php?term= Glasshole). Although research on digital platforms is challenging and there are many perspectives to look at to fully understand the complexities for a success or failure, taking an early end-user perspective to the google glass platform would probably have exposed these issues above at an earlier phase.

This human-centred perspective on digital platforms has been identified as one of six key focus areas on digital platforms within Information Systems research [8]. The authors ask the question "How do digital platforms affect everyday life?" and raises critical issues regarding what these digital innovations bring with it in terms of lack of transparency and privacy as well as a shift in the power relations between different stakeholders. They also point out that digital platforms 'support new ways of interacting within communities and through mediated cocreation' that are not yet taken into account of how people actually experience the integration of digital platforms into the social world.

A lot of digital platform research has tried to understand their impact on customers purchase intention based on customer experience [9], however the critical determinants of success in digital platform business models are still difficult to understand and unclear $[10,11]$.

While taking a traditional technology-oriented direction to digital platforms tends to become too busy in focusing on reducing cost or smoothing out the operational inefficiencies, a human-centred approach enables research to identify new user values or how technologies can be adapted to changing user needs [12]. The latter is one of the key routes for IS research on digital platforms according to de Reuver, Sørensen and Basole [8], and it is where we place our contribution with this article.

As EV's with their battery storage enable technical possibilities to be always connected, powered up and sharing energy, they open up for relations to new emerging digital platforms, which extend the end user's experiences of EV's. In the following sections we will discuss the challenges in understanding how digital platforms affect everyday life in relation to EV use and how to approach it.

\subsection{EVs making life digital}

As we have discussed above, research on digital platforms is challenging because of how they are distributed and intertwined with institutions, market and technologies and digital platform innovation spreads with increasing architectural complexity across many different industries. How the introduction of digital platforms changes traditional manufacturing industries has received a heightened attention within Information Systems (IS) research [13], and research has shown that traditional car manufacturers have not fully understood the transition to EVs and the relation to new digital platforms [3]. Some studies show examples of how EV owners have to adapt their driving routines and planning in order to manage the different digital technologies related to finding, connecting and paying for charging as there are many new charging service providers with different geographical spread, user interfaces and payment models [14, 15], which even led to some people abandoning EVs and returning to fossil fuel cars [16]. 
This emphasizes that planning and technology support are important aspects in transitioning to EV driving [15]. This has opened up for new companies to rethink EVs, not only to make charging experience better (https://cleantechnica.com/2020/11/09/tesla-ownersshare-supercharging-experiences-as-tesla-announcesits-20k-supercharger-milestone/), but also to innovate and provide new products and digital services around EVs. The common idea that runs through these innovations is to think about cars as more than means for transport, but also provide new possibilities for EVs as always connected and to be integrated into people's wider everyday life [17], e.g. as Vehicle to Grid (V2G) energy source [18], mobile surveillance [19], or as a mobile processing power source [20]. However, as EVs and the surrounding new evolving digital platforms are in a phase of rapid development, there is a lack of knowledge within academia and industry around how people integrate and anticipate their future life with EVs and relevant digital platforms [3].

\subsection{Understanding everyday digital life with EVs}

Reviewing current research on EV attitudes and automotive industry development on EV's expose a bias towards technology acceptance or product experience research and a gap in knowledge in understanding how EVs as an emerging new technology integrate in people's everyday life with digital technologies [2, 3, 21]. A major part of the EV experience related research has been focusing on challenges in EV adoption, acceptance or driving experience based on historical models and parameters. These approaches are not designed to foresight people's anticipation of future EVs, thus limiting the possibilities for co-creative digital platform innovation for the automotive industry. EV adoption models based on the technology acceptance model (TAM) [22] and individual EV user experience research [2], are now being developed through real life based approaches such as ethnography [15, 23]. In the paper we will demonstrate such an ethnographic approach enables new understandings of the wider context of how people actually learn to live with EVs and related digital platforms to identify the real challenges for the users and their anticipations of the future.

\section{Methods}

We have applied an ethnographic approach [24, 25 ] to be able to investigate challenges and opportunities with digital platforms connected to EVs.
Our findings presented in this article derives from an ongoing academic-industry collaborative research project about smart charging of EVs in private households, called RELEVANT. The project is part of the Swedish strategic innovation program for future sustainable mobility solutions (VINNOVA, award no: 2019-03065), that incorporates car manufacturing industry, energy providers, research institute and academia. Our findings are extracted from an ongoing 2-year study of people in ten private households with EVs (including both plug-in hybrids and full electric vehicles) whereas four of the households also produce energy through solar panels. Through the project, the research participants will be provided with a prototype of a digital platform for smart EV charging at their home. Part of the RELEVANT project outcome will be to develop a smart charging algorithm to optimize the time for charging depending on various sources such as collected data from various sources such as momentary household peak load, energy cost, vehicle usage, weather or user preferences. The ethnographic investigations of how these ten households learn to live with the digital platform provide insights to iteratively develop the charging function through the life of the project and better understand what smart charging actually mean for people in everyday life.

In this paper we present the findings from the first set of interviews with the families. These interviews focused on the research participants experiences and anticipation on EV use and smart charging before they were introduced to the smart charging function and any equipment installed in their homes. The ethnographic data was produced by using an openended interview guide and discussing each family's adoption and anticipation of the smart charging function through an online video call interview. These digital interviews were semi-structured group interviews with each household including all members that used the EV in the family. Each interview was led by one or two researchers and lasted for approx. 1 hour each.

Our first interviews had an explorative and broad scope to both get to know the participants in the households, their EV preferences, their everyday driving routines and EV experiences, their household energy awareness and strategies, and finally their anticipations on EV's and home energy management. The main theme in the interviews circled around what smart home technologies meant for them.

The video recorded interviews were then transcribed and analysed through thematic analysis [26] by two researchers. Totally 10 hours of interview transcripts were coded into different codes for thematic analysis, (e.g., "anticipation of charging", "unsmart technology", "changing routines" etc.) 
These codes were then analysed by two researchers and compiled into three themes explained in the next chapter of findings: experiencing digital life with EVs, learning to live with EV and Imagining future digital EV solutions.

\section{Findings}

Through our ethnographic approach we managed to identify key components of the transition the research participants experienced when they changed from fossil fuel cars to EV's, and how this transition proved to be much more than only switching fossil fuel for electricity. That is, the findings demonstrated how the participants' experiences and anticipations often were related to how they experienced the digital infrastructure in which the maintenance and caring of the EV was integrated, rather than experiences of the $\mathrm{EV}$ in itself. In the following we describe three dimensions of the impact of digital platforms had on the research participants everyday life with EVs according to our analysis of the ethnographic material produced through the group interviews. These three dimensions relates to different routes to integrating the EVs into everyday life; the direct experience of the digital life with EVs, what and how the families learned to live with EVs and finally how the families imagined how their everyday life would evolve through their experiences and learnings. Some of these households had plug-in hybrid vehicles with both electric and fossil fuel engines and some had fully electrified cars. We have anonymised our households A to $\mathrm{J}$ and reference them as $\mathrm{HA}-\mathrm{HJ}$ and the persons in the household as $\mathrm{F}=$ Father, $\mathrm{M}=$ Mother, $\mathrm{C}=\mathrm{Child}$, and $\mathrm{R}=$ Researcher.

\subsection{Experiencing digi-physical world of EVs}

In the interviews with the families of the households they often compared the previous life with fossil fuel cars with their current life with an EV. Many of the traditional performance aspects of cars, such as acceleration, road handling and noise were often seen as better for the EV compared to the fossil cars. However, when the research participants discussed their issues and challenges with their EVs, they referred to the new surrounding digital infrastructures around EVs. They described how the amount of new service providers, with different interfaces, payment models and processes to sign up for a service became challenging to understand and use in everyday life. Refuelling gas was seen as easy since they knew where the gas stations were located, whereas finding electrical chargers was often mentioned as more hidden and harder to find even if they probably could be searched online. The father in HF explained:

$\mathrm{HF}(\mathrm{R})$ : "Do you look up charging stations, do you use an app or...?"

$\mathrm{HF}(\mathrm{F})$ : "Yeah well sometimes but it's- I realize that it was quite hard, and they were quite few, and you had to look on so many different websites, so it was not really convenient to find charging."

At the same time, it was mentioned during the interview in HF, that since electricity is available almost everywhere, the number of possible places to charge could be even greater. However, the mother in HF mentioned the lack of transparency in how much it would cost to charge:

$\mathrm{HF}(\mathrm{M})$ : "But it is interesting with the electricity when you want to fuel up electricity, that's quite different, because now we can find it easily, but it costs quite a lot in some places."

Even if the electric power provided were free, the process to be able to start charging seemed to involve a lot of steps to become a registered user of the charging network. Like the father reflected in $\mathrm{HB}$ about the low usage of the free EV chargers at the local grocery market:

$\mathrm{HB}(\mathrm{F})$ : "That's my guess because I was surprised that not more people are using them because you need to order a card from [Grocery store] and its through [Energy company], one of the electrical companies, so it was a process to get this card and I think that's probably enough for some people that don't do it and then they try it and then 'oh it doesn't charge' and then they skip it."

The number of different charging providers seemed to increase the complexity to able to easily find, connect and pay for charging, as the father in $\mathrm{HC}$ explained:

$\mathrm{HC}(\mathrm{F})$ : "Very seldom I would say, in the areas where I normally park in the city there are no charging available so it's no charging stations available, but it has happened once in a while because I have this [Public charger provider] chip on the key when-and then I need to know if it is an [Public charger provider] charging station also so I- otherwise it's no use. I mean like if I go to the [Grocery store] to shop, they have the wrong charging card or whatever. it's called so I cannot use the [Public charger provider] that I have. So, it's- they-it's a little bit tricky this thing that we have so many different suppliers of charging stations. In principle you should have like five or ten different subscriptions to be able to utilize it fully. "

And even if taking all the work of signing up for a number of services, the fast evolvement of digital services and related hardware were sometimes mentioned as hurdles to be too fast to jump on a new 
digital service, as the father in $\mathrm{HC}$ explained why they felt unsmart:

$\mathrm{HC}(\mathrm{F}):$ :Mmm ... The biggest problem I would say with the new smart technology is that you probably must change it after a few years. Either because its obsolete or because it breaks. I mean the development is not linear any longer and when... if you would like to have something new and you need to have something that's compatible to something that is 2-3-4 years old, and then you have to reinvest again in the same thing."

The experience of the new digital life with EVs explained by our households, exposed the digital platforms involved as much more abstract and more complex to understand than the concrete life of traditional car ever was. Thus, living with fossil cars was expressed as a more analogue and tangible experience in comparison. You fuelled up your vehicle at specific places (gas stations) and paid with your own credit card to your preferred gas provider for a specific amount of gas. As digital infrastructures and services to its nature could combine many actors, data, business models and transform rapidly, it becomes black boxed for the end user's. The sometimes untransparent and complex structure of energy providers, power grid distributors, payment models and automotive companies takes a lot of effort to understand. However, even if the user does not bother to understand all relations within the black box, their willingness to go through a long complex process of signing up could easily become the main argument for not starting to use a digital service.

Also, the fast evolvement of different digital services makes them avoid jumping on too many new services to avoid the risk of keeping updating things all the time.

\subsection{Learning to live with EVs}

Even digital life with an EV provided the households with a lot of obstacles to select and join the relevant charging services for them, it was however seen as something you learnt to deal with as many other digital things in their lives. This led us to the understanding that one dimension of integrating EVs into everyday life is in fact to appropriate the digital technologies the maintenance and care of the EV brings with it. For example, in the daily travel routines, the mother in $\mathrm{HJ}$ explained how she only selected the most necessary services from a small fraction of available providers and don't waste time for others: $\mathrm{HJ}(\mathrm{M})$ : "Ehm... As it is today, I charge at [Workplace] and I charge at home... so it takes me half of the trip with battery and half of the trip with the fuel. And to be honest, I have put it on charging in [City centre] area as well. But it's a lot of applications you must download, so I also have... I think, three different charges [Charging providers] as it is now. And when I try to charge it at another solution, so you have to sign up...it's too many charging options as it is. So, if it is one of them, I have, I use it. Otherwise, I don't give a shit."

She continued to explain how also adding a physical card or tag to pay for charging was making the charging experience easier and more stable than through the charging providers smartphone app:

$\mathrm{HJ}(\mathrm{M})$ : "I think I use the card, as it is just now. Because it is quicker, I have it in my door pocket, so I can just *swipe it*. And it's logged on, so that's easier. You can take it here, but it's a lot of steps here. You have to find the map, and you have to find the... and then you have to click on the number, so you have to... So, I think it's easier with the card. So quick, it has to be like that."

The stability of digital services was also mentioned as a problem where it sometimes was hard to understand the reason for a temporary failing service. For example, the father in HG referred to how difficult it was to understand and fix the problem when the TV streaming service at home failed:

$\mathrm{HG}(\mathrm{F})$ : "No, but it works 99 out of 100 . Then you know from having experience from several streaming services, it is like this. The first thing they blame your internet provider, but in reality, it's their streaming service that has bad functionality for a time period and makes it impossible to connect. That's the problem, you have so many boxes that could cause the problem. And you don't know where to start. It could be the router that has to be restarted, or the TV, or the streaming service, or..."

If trying to solve these kinds of issues without any past experience often led to searching the internet for tips and tricks, rather than calling any IT support, like the father in $\mathrm{HC}$ explained:

$H C(F)$ : "Yeah I mean it depends on what is it of course. In some cases, I think I have the knowledge to solve it by myself and if I don't have the knowledge there's something called Google, I think it is, or you read the instruction manual or whatever it is and then you fix it. Itsyeah, I would say it's very very seldom that we bring in someone, some IT-technician or something in the house. We have never done that."

As discussed above, learning to live with new digital services, was often referred to moments of making the digital more tangible. The father in HI described how he was exploring and pre-planning where he would stop and charge during his business trips stops (driving a fossil fuel company car): $\mathrm{HI}(\mathrm{F})$ : "I think it's more, more... Common with charge stations. I just now stopped where I 
always stop, in [City far from home]. And they have... a new station for electricity charging. For I think it's five positions, where they can quickly charge, only one or two months old. it's really nice, and there was one car [charging] when I arrived. And I think it will be more and more like that, it has to be more and more like that, of course. If it should be more convenient to use the car... So... So then if I should do the trip, I do now, to [City for long business trips], which is normal. It should be perfect to stop here and charge, and I can go on to [City for long business trips] and continue my journey."

Learning to live with the digital infrastructures around EVs created a lot of effort to understand and start to use the services that you needed for your EV life, however the effort needed to do this change was somewhat expected as it could be referred to the general transition into digital services in their everyday life. To be able to handle the abstractness of digital services, the households seemed to use strategies of only selecting the most necessary services, making the digital more tangible by learning where the charging places were localised and shifting to payment methods where physical cards or tags made the process of starting and paying for $\mathrm{EV}$ charging more tangible. Solving problems when public charging fails, could be difficult as the number and types of errors is almost impossible to overview for an end user. Learning from others seemed to be best done through googling on the internet.

\subsection{Imagining future digital EV solutions}

The third dimension of integrating EVs into everyday life had to do with the emerging expectations and anticipations the research participants articulated when getting familiar with the EVs and learning to live with the digital platforms the EVs were integrated with. These expectations and anticipations related to how they imagined their future use of their EV and extension of what an EV could be used for more than means of transport. This was expressed by the mother and the two teenagers in HA when they discussed how the future charging services should be combined with other services to make the charging stop more entertaining or efficient.

$\mathrm{HA}(\mathrm{M})$ : "No, but it would be very nice if the public chargers are placed at shopping malls or something else, where you could do something. Then you could do some errands at the same time as you charge the car, like you combine charging when doing grocery shopping..."

$\mathrm{HA}(\mathrm{C} 1)$ : "We could do...like an idea I have come up with. I know that Apple is doing route planning and if you plan a route then you could choose 'Eco' when you drive on electric, then I know that they have a solution showing you were to charge and then what you can do around the charge spot."

HA(C2): "It's like it knows, it chooses charging stations and then you get help to plan."

$H A(M)$ : "It becomes sort of a new type of lifestyle"

Connecting other digital services to charging services were not the only thing that inspired the households; ideas of how the EV could be connected to the household's energy management system were seen as great potentials for making life less complicated and more sustainable. For example, the father in $\mathrm{HH}$ explained how he anticipated that a smart algorithm could make EV charging at home more energy efficient without the need for changing their routines:

$\mathrm{HH}(\mathrm{F})$ : "In our case I see mainly advantages to having an algorithm to make it energy efficient. Just because you are busy with work, activities and other things. It would be good to come home and simply plug it in. Knowing that it will charge when there is less load on the grid, I suppose is feasible. Then also that it maybe knows you're driving routines and how much energy is needed, so it could balance in that way. E.g., which days it needs to be fully charged and when it's not..."

The battery of the EV appeared to play an important part of the future smart household energy management system, however the priorities of which energy consumers to be most important at the moment might differ depending on the everyday activities to avoid unexpected delays. This was explained by the father in HD:

$\mathrm{HD}(\mathrm{F})$ : "What I believe and want to see in this project is a smart management of the energy consumption for the household. Like I as a customer don't have to care so much about, if the heating system needs to start, it has priority to the dishwasher or charging your car... You want to optimise the possibility of charging when it's cheaper, however the household should have priority so if you go down in the basement you don't want to find out that the washing machine has stopped."

Finally, the father and mother in $\mathrm{HH}$ concluded the importance for the EV to be able to be compatible, connect and evolve with these different digital infrastructures at home:

$\mathrm{HH}(\mathrm{F})$ : "Yes, I believe it's like, we talked about it at work as well, it will be crucial for companies like [car company] and the other, that the car is smart enough to help the customer to find the right charging place and payment for all the parts that are complicated.... That type of functionality will be absolutely necessary for 
you to be able to exchange both cars at home [to EV's]"

$\mathrm{HH}(\mathrm{M})$ : "Yes I agree. You want to be sure that you can drive around in an easy way."

In our interviews, the EV was often anticipated to be part of the future energy management system in society, in our interviews which shifted the focus from the traditional driving performance aspects of the car to become a piece of a puzzle in a future energy ecosystem and its capabilities to work seamlessly with future digital services related to electric energy.

\section{Discussion}

In our work, our ambition has been to contribute to the field of research on digital platforms by ethnographic investigations how the digitalisation of cars through digital platforms can be understood from social perspectives. Our findings exemplify how ethnography can be helpful to explain the possibilities and challenges that people are facing and dealing with when changing from fossil fuel vehicles to EV in real life settings. Below we will discuss these findings from three different perspectives; In how EVs transform traditional analogue car experiences into a more digital world; how a research perspective of everyday life with digi-physical EV experience can share light on end user experiences and anticipations on related digital platforms; and how ethnographic findings provide implications for the automotive industry to understand EVs relation to these digital infrastructures from their user's perspective.

\subsection{EVs turns analogue into digital world}

Our study identified several narratives from our participants about how it felt to become an EV owner from previously driving a fossil fuel car. For the participants it was not only about exchanging the petrol to electric energy and continuing with the same driving habits and car maintenance routines. Our findings showed that fossil fuel use could be referred to as a more "analogue" experience where you refuel at specific gas stations, with an almost fixed price per unit, payment through cash or credit card. The fossil fuel is pre-defined only for driving at an almost fixed consumption rate per distance. When comparing their new experience with an EV, it became apparent that living with an EV is extending the experience of caring for and maintaining a car into a digital world. As they had the possibility to conveniently charge at home the car became connected to their home energy system, meaning that they were paying for energy through their household energy bill. However, as the energy price could be variable hourly according to the spot price on the energy market it also became very difficult to fully understand the "fuel" costs of the car.

The extension into digital car owning experiences also became apparent in our study when the research participants talked about their experiences trying to use public charging. To find the right type of charger for your EV, understand the various energy tariffs and pay for charging and parking, forced the research participants to download several different apps, create login accounts, connect payment preferences and actively plan their routine according to the approximated charging speed. Even if the participants did not currently have the technical possibility to the use the energy in the battery for other than things than driving, some of them already anticipated possibilities to use the car's battery as an energy storage for their solar panels on their house, back-up for power out in the grid or even being able to trade electric energy by charging when energy was low and sell when price is high. This would mean new connections to digital platforms for energy trading.

This connection of EV's to various new digital platforms expose a lot of new opportunities for new services and choices for the end users, however the large amount of new digital platforms [27, 28] and uncoordinated relations between them provided confusion and complexity into everyday life. "Range anxiety" [29] was not the important topic in our study, but rather "charge anxiety" of being able to do public charging. Digital experiences around EVs need to be more transparent, connected and seamless in order to avoid the struggles for the EV users. Thus, our study identified people's anticipatory experiences of EVs to be more than simply exchanging the fuel pump for a socket plug, but instead imply a transition from a tangible analogue world with fossil fuel cars into an abstract and complex situation that straddles the digital and physical components of EV use.

\subsection{Perspective of everyday life}

The transition from an analogue to a digi-physical world became evident through our ethnographic understandings of people's experiences and expectations of EVs. As in our earlier reference to the Google glass story [7] there are reasons to believe that the transition into electric vehicles may fail to some degree if the digital platform component in EV use is not acknowledged in user research and technical development. Indeed, the end-user's early responses to products and services of a digital platform are key for failure or success whatever the technical solution or business model looks like in theory [9]. 
Through our ethnographic investigations [24], the details of the everyday life struggles with digital technologies or anticipation of the future possibilities were exposed. Through ethnography such small details as needing to create yet a new login account for a digital service around charging expose how this not only was related to the specific service, but also to a general opinion that there were not "worth the fuzz" to charge public in many situations. However, our ethnographic findings did not limit us to explain current experiences of EVs, but also more futureoriented insights into how the participants anticipated the future use and expectations of features of new digital platforms making sense for their everyday EV routines. As some participants anticipated a future with an EV as energy storage with the possibility for them to use as they want to increase their independence, ethnography manages to foresight details of their anticipations of a future digital technology solution and connect to their higher values in life. Through ethnographic methods, the relation and compatibility between different digital platforms from an end-user perspective becomes visible through the narratives of the participants when they describe their everyday messiness in both trying to pay for both charging and parking at the same time to different digital services.

Interestingly, even if an ethnographic approach are not new to the field of IS [30], its use in understanding digital platforms seems rare although customer experience and hedonic factors are found to be important for a success of a digital platform service [9]. Ethnography fits well to address the question of "How do digital platforms affect everyday life?" [8] by providing valuable insights of customer experience from an end-user perspective in a complex digiphysical world.

These findings were based on the first results from our smart charging project, and we see potential in involve the participants in iteratively co-creating the smart charging platform based on our ethnographic insights in the research participants' EV experiences and anticipations.

\subsection{EVs as part of evolving digital infrastructures}

Our findings demonstrate how electrification and digitalisation of cars provide new foresights in what is important to people in everyday transport and logistics today and in the future. Based on the narratives from our participants transition from an analogue to a digiphysical world through EVs, we can conclude that automotive industry and academia need to expand the scope of investigations outside the physical in-car context and look beyond the comparison with the fossil fuel vehicle experiences as a starting point. As the development of EV's move swiftly from analogue to digi-physical experiences for the users, it's important to as early as possible identify what this means in terms of everyday life hazzle and as well as opportunities. In doing so, the risk of creating incompatibilities between the EV and relevant digital infrastructures is mitigated. Instead, studies of how people appropriate the digi-physical components of EVs provide context for creating meaningful digital services as well as opportunities for digital platform innovation. Our findings indicate that the technical performance of an EV becomes secondary for a user if it's not fully compatible with surrounding digital environment e.g., when the user is struggling to understand where there's an available and working charge point for their EV, which service provider, the cost and time for charging and what other services close by to spend time at while waiting.

We also found transformative aspects of EVs to facilitate an increasing knowledge around energy and energy usage of the participants [23]. This generated emerging needs and ideas from our participants in being able to use their EV as part of the home energy system to become more independent in balancing their energy usage to avoid peak costs, power outs or trading energy. These anticipations foresight EVs to become part of an increasing number of emerging digital energy platforms $[31,32]$. Subsequently, the automotive industry needs to take into account that EV's are more than a means for transportation, they are also part of emerging digital energy platforms. Our findings emphasize the extension in how digital infrastructures have an increasing part of the future car experience and difficulties in separating a car driving experience from its digital context, an insight that might have implications for future user experience research on EVs.

To pragmatically address the user issues and anticipations from this study, we suggest that the automotive industry give more attention to EVs current and future surrounding digital infrastructures. Our results indicates that seamless integration with various digital platforms is key to support the appropriation of EVs in society. Another key component in successful implementations of EVs might be to include users into co-creative development processes to incorporate user's evolving anticipations of future use. Our ethnographic approach and the insights into users' anticipatory experiences demonstrate how such an approach could enable the first step in this process. The digital transformation for the automotive industry is not about turning the car into a digital product, it's about making the car part of 
new emerging digital infrastructures and making it possible to evolve with the users in order to stay relevant.

\section{Conclusions}

We have presented ethnographic findings from an empirical study of ten households with privately owned EVs and explored the impact of digital platforms related to their EV use. We explored EVs and related digital platforms from an end-user perspective by taking an ethnographic approach to answer our question in how to understand the impact digital platforms related to EVs have on everyday life and implications for digital transformation of the car industry. Our empirical findings are based on the first study of a longer project on developing a smart charging platform for EV charging at home with the participants.

Our main conclusions are: Firstly, the use of EVs is coupled with a multitude of digital platforms previously not connected to car ownership and driving. These new digital infrastructures around EVs enable new future possibilities for the users to use their $\mathrm{EV}$ for more than means of transport. However, today the multitude of different digital services with uncoordinated systems provide a lot of struggles for the end-user in being able to find, connect and pay for charging. Secondly, an ethnographic approach to understanding EVs and digital infrastructures, does not only explain and uncover experiences of appropriating how to plan and live with an EV within its digi-physical context of both digital platforms and physical charging stations, but also enables foresighting into EV futures through the human centric perspectives to digital platforms integrated in EV use. Finally, this has implications for how automotive industries need to develop their understanding of how electrification of cars changes what it means to use a car in everyday logistics in terms of how it becomes part of wider digital infrastructures.

\section{References}

[1] IEA. Global EV Outlook 2020 - Entering the decade of electric drive? In (June 2020). Retrieved 2021-05-01 from https://www.iea.org/reports/global-ev-outlook2020

[2] Edmond Daramy-Williams, Jillian Anable, and Susan Grant-Muller. 2019. A systematic review of the evidence on plug-in electric vehicle user experience. Transportation Research Part D: Transport and Environment 71(2019/06/01/), 22-36. http://dx.doi.org/https://doi.org/10.1016/j.trd.2019.01. $\underline{008}$
[3] Michael K. Svangren, Mikael B. Skov, and Jesper Kjeldskov. 2017. The connected car: an empirical study of electric cars as mobile digital devices. In MobileHCI '17: Proceedings of the 19th International Conference on Human-Computer Interaction with Mobile Devices and Services ACM, Vienna, Austria, 1-12. http://dx.doi.org/10.1145/3098279.3098535

[4] Axel Schmidt, Teodoro Lio, Juergen Reers, and Andrea Regalia. The Electric Vehicle: Why Manufacturers need to rethink their ecosystems, the customer journey, and the enitre value chain. In (Jan 18, 2021). Retrieved 2021-06-10 from https://www.accenture.com/usen/insights/automotive/electric-vehicle-more-newpowertrain

[5] Jie Deng, Chulheung Bae, Adam Denlinger, and Theodore Miller. 2020. Electric vehicles batteries: requirements and challenges. Joule 4, 3, 511-515.

[6] Lance Noel, Gerardo Zarazua de Rubens, Benjamin K. Sovacool, and Johannes Kester. 2019. Fear and loathing of electric vehicles: The reactionary rhetoric of range anxiety. Energy Research \& Social Science 48(2019/02/01/), 96-107. http://dx.doi.org/https://doi.org/10.1016/j.erss.2018.10 .001

[7] Amarolinda Klein, Carsten Sørensen, Angilberto Sabino de Freitas, Cristiane Drebes Pedron, and Silvia Elaluf-Calderwood. 2020. Understanding controversies in digital platform innovation processes: The Google Glass case. Technological Forecasting and Social Change 152(2020/03/01/), 119883. http://dx.doi.org/https://doi.org/10.1016/j.techfore.201 $\underline{9.119883}$

[8] Mark de Reuver, Carsten Sørensen, and Rahul C. Basole. 2018. The Digital Platform: A Research Agenda. Journal of Information Technology 33, 2, 124135. http://dx.doi.org/10.1057/s41265-016-0033-3

[9] Fatemeh Saberian, Mirahmad Amirshahi, Mahdi Ebrahimi, and Asieh Nazemi. 2020. Linking digital platforms' service dimensions to customers' purchase. The Bottom Line 33, 4, 315-335. http://dx.doi.org/10.1108/BL-01-2020-0001

[10] David P McIntyre and Arati Srinivasan. 2017. Networks, platforms, and strategy: Emerging views and next steps. Strategic Management Journal 38, 1, 141160.

[11] Margherita Pagani. 2013. Digital business strategy and value creation: Framing the dynamic cycle of control points. MIS Quarterly, 617-632.

[12] Hal R. Varian. 2010. Computer Mediated Transactions. American Economic Review 100, 2, 1-10. http://dx.doi.org/10.1257/aer.100.2.1

[13] Marin Jovanovic, David Sjödin, and Vinit Parida. 2021. Co-evolution of platform architecture, platform services, and platform governance: Expanding the platform value of industrial digital platforms. Technovation(2021/01/10/), 102218. http://dx.doi.org/https://doi.org/10.1016/j.technovation .2020 .102218

[14] Xin He, Dannuo Jiao, Jinghan Xu, and Ling Wang. 2019. Innovative ideas for charging piles based on existing problems for new energy vehicles. IOP 
Conference Series: Earth and Environmental Science 300(2019/08/09), 042068. http://dx.doi.org/10.1088/1755-1315/300/4/042068

[15] Rikke Hagensby Jensen, Michael Kvist Svangren, Mikael B Skov, and Jesper Kjeldskov. 2019. Investigating EV Driving as Meaningful Practice. In 31th Australian Conference on Computer-Human InteractionConference of the Australian ComputerHuman Interaction Association for Computing Machinery.

[16] Scott Hardman and Gil Tal. 2021. Understanding discontinuance among California's electric vehicle owners. Nature Energy 6, 5 (2021/05/01), 538-545. http://dx.doi.org/10.1038/s41560-021-00814-9

[17] Dakota LD Nicley, Edward J Lazaros, Allen D Truell, Jensen J Zhao, and Christopher B Davison. 2020. THE CONNECTED CAR: A GLIMPSE INTO THE FUTURE OF TRANSPORTATION. Issues in Information Systems 21, 2.

[18] Willett Kempton and Jasna Tomić. 2005. Vehicle-togrid power implementation: From stabilizing the grid to supporting large-scale renewable energy. Journal of Power Sources 144, 1 (2005/06/01/), 280-294. http://dx.doi.org/https://doi.org/10.1016/j.jpowsour.20 04.12 .022

[19] Shaomin Xiong, Toshiki Hirano, and Neale Jones. 2020. A Low Power Smart Security Surveillance System for Vehicles. In ASME 2020 29th Conference on Information Storage and Processing Systems. http://dx.doi.org/10.1115/isps2020-1948

[20] Lewis Tseng, Takamasa Higuchi, and Onur Altintas. 2017. When cars meet distributed computing: Data storage as an example. arXiv preprint arXiv:1711.02014.

[21] Virender Singh, Vedant Singh, and S. Vaibhav. 2020. A review and simple meta-analysis of factors influencing adoption of electric vehicles. Transportation Research Part D: Transport and Environment $\quad 86(2020 / 09 / 01 /), \quad 102436$. http://dx.doi.org/https://doi.org/10.1016/j.trd.2020.102 $\underline{436}$

[22] Fred D Davis. 1985. A technology acceptance model for empirically testing new end-user information systems: Theory and results Massachusetts Institute of Technology, Cambridge, MA.

[23] Marianne Ryghaug and Marit Toftaker. 2014. A transformative practice? Meaning, competence, and material aspects of driving electric cars in Norway. Nature and Culture 9, 2, 146-163. http://dx.doi.org/10.3167/nc.2014.090203

[24] Karen O'Reilly. 2012. Ethnographic methods. London: Routledge, 2012.

[25] Jay Hasbrouck. 2017. Ethnographic Thinking: From Method to Mindset. Routledge.

[26] Virginia Braun and Victoria Clarke. 2006. Using thematic analysis in psychology. Qualitative Research in Psychology 3, 2 (2006/01/01), 77-101. http://dx.doi.org/10.1191/1478088706qp063oa

[27] Y. L. Zhukovskiy, P. K. Suslikov, E. G. Arapova, and L. Z. Alieva. 2020. Digital platform as a means of process optimization of integrating electric vehicles into electric power networks. Journal of Physics: Conference Series 1661(2020/11), 012162. http://dx.doi.org/10.1088/1742-6596/1661/1/012162

[28] Omar Isaac Asensio and Sarah E Walsh. 2018. Mobile Apps for Workplace Charging: A Big Data Field Experiment in Electric Vehicles. Academy of Management Global Proceedings Surrey, 2018, 208. http://dx.doi.org/10.5465/amgblproc.surrey.2018.0208 . $\mathrm{abs}$

[29] Nadine Rauh, Thomas Franke, and Josef F. Krems. 2015. User experience with electric vehicles while driving in a critical range situation - a qualitative approach. IET Intelligent Transport Systems 9, 7, 734739. $\quad$ http://dx.doi.org/https://doi.org/10.1049/ietits.2014.0214

[30] Michael Myers. 1999. Investigating information systems with ethnographic research. Commun. AIS 2, 4es, 1.

[31] Florent Grée, Vitaliia Laznikova, Bill Kim, Guillermo Garcia, Tom Kigezi, and Bo Gao. 2020. Cloud-Based Big Data Platform for Vehicle-to-Grid (V2G). World Electric Vehicle Journal 11, 2, 30.

[32] Tobias Menzel and Timm Teubner. 2020. Green energy platform economics-understanding platformization and sustainabilization in the energy sector. International Journal of Energy Sector Management. 